\title{
A Lowering of Breast and Ovarian Cancer Risk in Women with a BRCA1 Mutation by Selenium Supplementation of Diet
}

\author{
Tomasz Huzarski', Tomasz Byrski', Jacek Gronwald', Elizbieta Kowalska', Stanisław Zajączek', Bohdan Górski', \\ Jowita Huzarska', Cezary Cybulski', Steven A. Narod', Jan Lubiński' \\ IInternational Hereditary Cancer Center - Department of Genetics and Pathology, Pomeranian Medical University, Szczecin, Poland; 2Women's College Hospital, \\ Toronto, Canada
}

Key words: selenium, BRCA1, cancer prevention

Corresponding author: Tomasz Huzarski, International Hereditary Cancer Center, Pomeranian Medical Univeristy, ul. Połabska 4, 70-115 Szczecin, Poland; e-mail: huzarski@sci.pam.szczecin.pl

Submitted: 20 December 2005

Accepted: 10 January 2006

It has been shown that individuals with inherited predisposition to cancer (including colon, breast and ovary) have increased sensitivity to bleomycin. We published that bleomycin-induced chromosomal instability in BRCA1 carriers is inhibited by selenium supplementation in physiologic (according to $\mathrm{WHO}$ ) doses. Selenium was shown to reduce the risk of several cancers.

The aim of our study is to verify the idea that selenium supplementation of diet reduces the risk of cancer in women with a BRCAl mutation. We performed two pilot studies involving 200 healthy BRCA1 mutation carriers (100 matched pairs - cases and controls). After two years of oral selenium administration the frequency of BRCA 1 -associated tumours was two times lower in women who supplemented their diet with selenium, as compared to women without supplementation.

We decided to verify these results in a clinical trial of a large series of BRAC 1 positive women. This project includes 1,800 BRCA1 carriers aged older than 25, healthy or after unilateral mastectomy because of breast cancer. All women supplement their diet with selenium solution (Sel Vita Gen) produced by Vifarm S.A. in Warsaw. Fourteen centres throughout Poland participated in this study. Until December 2005 the study included 1,469 women with the BRCA1 mutation. Now, 960 women have regular supplementation with
Sel Vita Gen and these are seen every 6 months for control visits (clinical examination and analysis of serum selenium level). 509 do not have supplementation because of pregnancy, bad toleration, or withdrawal of their participation.

After 18 months of this study we reported 8 new cases of cancer in healthy women (5 breast cancers, 2 ovarian duct cancers, 1 ovarian cancer) and 8 cancer cases in women after mastectomy (5 breast cancers, 3 ovarian cancers). In order to obtain reliable results we will continue this study until we diagnose at least 60 new cases of cancer. 